\title{
Dronedarone for atrial fibrillation: a new therapeutic agent
}

This article was published in the following Dove Press journal:

Vascular Health and Risk Management

4 August 2009

Number of times this article has been viewed

\author{
Pawan D Patel \\ Rohit Bhuriya \\ Dipal D Patel \\ Bhaskar L Arora \\ Param P Singh \\ Rohit R Arora \\ Department of Cardiology, \\ Chicago Medical School, \\ Chicago, IL, USA
}

Correspondence: Rohit Arora

Department of Cardiology, Chicago Medical School, North Chicago VA Medical Centre-I33B, 300 I Green Bay

Road, North Chicago, IL-60064, USA

Tel + I 2246104503

Fax +I 2246103878

Email rohit.arora@va.gov

\begin{abstract}
Atrial fibrillation is the most common of the serious cardiac rhythm disturbances and is responsible for substantial morbidity and mortality. Amiodarone is currently one of the most widely used and most effective antiarrhythmic agents for atrial fibrillation. But during chronic usage amiodarone can cause some serious extra cardiac adverse effects, including effects on the thyroid. Dronedarone is a newer therapeutic agent with a structural resemblance to amiodarone, with two molecular changes, and with a better side effect profile. Dronedarone is a multichannel blocker and, like amiodarone, possesses both a rhythm and a rate control property in atrial fibrillation. The US Food and Drug Administration approved dronedarone for atrial fibrillation on July 2, 2009. In this review, we discuss the role of dronedarone in atrial fibrillation.
\end{abstract}

Keywords: dronedarone, amiodarone, atrial fibrillation

\section{Introduction}

Atrial fibrillation (AF) is the most common of the serious cardiac rhythm disturbances and is responsible for substantial morbidity and mortality. The prevalence of AF is almost $1 \%$ and it is estimated that by the year 2050, approximately 5 million subjects will have AF in the United States. ${ }^{1}$ Currently, there are two major treatment strategies for AF: rate control and rhythm control. Sustained sinus rhythm is associated with an improved quality of life and improved exercise performance. ${ }^{2}$

\section{Rationale for dronedarone}

Currently amiodarone is one of the most widely used and most effective antiarrhythmic agents $^{2}$ with little proarrhythmic potential. ${ }^{3}$ But during chronic usage amiodarone and its active metabolite desethylamiodarone can cause some serious extra cardiac adverse effects, including effects on the thyroid. ${ }^{2}$ Therefore there is a need for the development of safer and effective antiarrythmics agents. Amiodarone is an iodinated benzofuran derivative and contains $37 \%$ of organic iodine by weight. Amiodarone itself and its high iodine content can cause subclinical to overt thyroid dysfunction, manifesting either amiodarone-induced hypothyroidism or amiodarone-induced thyrotoxicosis. About $15 \%$ to $28 \%$ of patients develop thyroid dysfunction after 2 to 3 years of therapy, a risk that increases with higher doses. Moreover, amiodarone is lipophilic because of its iodine content, thus accumulates in adipose tissue and highly perfused organs like the liver, lung, cornea, and skin. In addition, because of its long half-life of up to 100 days, high iodine levels persist for $>6$ months after discontinuation of the drug. ${ }^{4}$ 
Dronedarone is a newer benzofuran derivative with a structural resemblance to amiodarone, with two molecular changes: it lacks the iodine moiety and it has a methane sulfonyl group that decreases lipophilicity, resulting in a shorter half-life and lower tissue accumulation. In vitro the amiodarone metabolite N-desethylamiodarone (DEA) strongly inhibited $\mathrm{T}_{3}$ binding to thyroid hormone receptor (TR), both $\mathrm{TR} \alpha_{1}$ and $\mathrm{TR} \beta_{1}$, within the same order of magnitude, whereas the active metabolite of dronedarone $\mathrm{N}$-debutlydronadarone (DBD) was shown to inhibit $\mathrm{T}_{3}$ binding to TR $\alpha_{1}$ but much less so to TR $\beta_{1}$. This isoform selectivity may explain the effects of dronedarone on the heart (a mainly $\mathrm{TR} \alpha_{1}$ organ) and the lack of effect on the liver (a mainly TR $\beta_{1}$ organ) with little effect on plasma thyroid hormone. ${ }^{5}$ The steady-state serum level of dronedarone is achieved in 5 to 7 days. The elimination half-life of dronedarone is 24 hours. It is cleared by nonrenal mechanism. Like amiodarone, oral bioavailability is increased 2- to 3 -fold when taken with food. It is well absorbed after oral administration (70\% to $100 \%)$. The bioavailability is relatively low (15\%) because of extensive hepatic first-pass metabolism by cytochrome P450 (CYP) 3A4 and CYP2D6, thus requiring twice-daily dosing to achieve steady-state serum levels. ${ }^{4}$

Dronedarone has been shown to exhibit even less reverse use-dependency of repolarization than that found with amiodarone, which may provide better cardiac safety. ${ }^{6}$ In patch-clamp experiments in canine ventricular myocytes, $10 \mu \mathrm{M}$ dronedarone markedly reduced the rapid component of the delayed rectifier potassium current $(97 \%, P<0.05)$ and the L-type calcium current $(76.5 \%, P<0.05)$. In the same experimental study on dog myocytes, acute superfusion of dronedarone shortened the APD in Purkinje fibres (at $1 \mathrm{~Hz}$ from $309.6 \pm 11.8$ to $287.1 \pm 10.8 \mathrm{~ms}, P<0.05$ ) and reduced the incidence of early and delayed after depolarizations induced by dofetilide and strophantidine in Purkinje fibers. Chronic treatment with dronedarone for 4 weeks, unlike chronic administration of amiodarone, did not lengthen significantly the QTc interval of the electrocardiogram. ${ }^{7}$

In multicellular preparations of guinea pig ventricular cells, dronedarone $(3,10$, and $30 \mu \mathrm{M})$ decreased maximum rate of rise of action potential $(\mathrm{dV} / \mathrm{dt} \max )$ with a concentration- and frequency-dependent relationship. ${ }^{8}$ Similarly, under whole-cell patch clamp on human atrial myocytes, amiodarone inhibited I ( $\mathrm{Na}$ ) by only $41 \%$ at $3 \mu \mathrm{M}$ while dronedarone inhibited I (Na) almost completely, by $97 \%$ at $3 \mu \mathrm{M} .{ }^{9}$ Thus dronedarone inhibits I $(\mathrm{Na})$ significantly in single human atrial cells and exhibits class I antiarrhythmic properties.
In patch-clamp technique guinea pig atrial cell, dronedarone inhibited the muscarinic acetylcholine receptoroperated $\mathrm{K}^{+}$current $\left(\mathrm{I}_{\mathrm{K}(\mathrm{ACh})}\right)$ either by depressing the function of $\mathrm{K}_{\mathrm{ACh}}$ channel itself or associated with GTP-binding proteins. Compared with amiodarone, dronedarone is an approximately 100 times more potent inhibitor on $\mathrm{I}_{\mathrm{K}(\mathrm{ACh})}$. This property may be involved, at least in part, in the antiarrhythmic action of dronedarone against $\mathrm{AF}$, as vagal activation plays a role in the pathophysiology of AF. ${ }^{10}$

Dronedarone is a multichannel blocker, as it decreases delayed-rectifier $\mathrm{K}^{+}$current $\mathrm{I}(\mathrm{Kr})$, slowly activating delayed-rectifier $\mathrm{K}^{+}$current $\mathrm{I}(\mathrm{Ks})$, and inward rectifier potassium current I (K1), L-type $\mathrm{Ca}^{2+}$ current I (Ca (L)) and maximum rate of rise of action potential ( $\mathrm{dV} / \mathrm{dt} \max )$ with a concentration- and frequency-dependent relationship (I (Na)). ${ }^{7,8}$ Thus dronedarone, like amiodarone, possesses both rhythm and a rate-control properties as it has class I to IV antiarrhythmic activity.

\section{Antiadrenergic property}

In anesthetized, atropinized dogs, both amiodarone and dronedarone were shown to inhibit alpha-adrenoceptor (adrenaline)-induced increase in blood pressure to similar extents. In the same study, inhibition of beta 1-adrenoceptor (isoprenaline)-induced increase in heart rate was less marked in dronedarone than amiodarone, whereas dronedarone was more potent than amiodarone in inhibiting beta 2-adrenoceptor (isoprenaline)-induced increase in blood pressure. However, in conscious dogs, both dronedarone and amiodarone inhibited isoprenaline-induced increases in heart rate by approximately the same amount. Thus, like amiodarone, dronedarone can partially inhibit the effects of stimulation of the adrenoceptor system that may play a pivotal role in the onset of severe ventricular rhythm disturbances. ${ }^{11}$ In a study on conscious dogs with healed myocardial infarction, dronedarone displayed antiadrenergic actions comparable to those of amiodarone. Both dronedarone and amiodarone significantly reduced the exercise-induced tachycardia and, at the highest dose, decreased the isoproterenol-induced tachycardia and did not impair the resting left ventricular function. ${ }^{12}$

Preclinical studies suggest that dronedarone is more efficacious in reducing ventricular arrhythmias. In a rat model, during a 20-minute period of ischemia, dronedarone significantly reduced the incidence of ventricular fibrillation (VF) from $80 \%$ to $30 \%(P<0.05)$ at $3 \mathrm{mg} / \mathrm{kg}$ and eliminated $\mathrm{VF}$ and mortality at $10 \mathrm{mg} / \mathrm{kg}$. In contrast, amiodarone at $10 \mathrm{mg} / \mathrm{kg}$ reduced significantly only the incidence of mortality during ischemia (from $60 \%$ to $0 \%, P<0.01$ ), 
while having no significant effect on $3 \mathrm{mg} / \mathrm{kg}$. On reperfusion (after a 5-minute period of ischemia), dronedarone reduced significantly the incidence of mortality (from 90 to $20 \%$, $P<0.01)$ at $1 \mathrm{mg} / \mathrm{kg}$ and eliminated VF and mortality when administered at 3 and $10 \mathrm{mg} / \mathrm{kg}$. Amiodarone (10 mg/kg iv) eliminated completely reperfusion-induced VF and mortality while having no significant effect at 1 and $3 \mathrm{mg} / \mathrm{kg} .{ }^{13} \mathrm{Simi}-$ larly in anesthetized pigs, dronedarone was more potent than amiodarone in reducing ischemia-induced ventricular arrhythmias. ${ }^{14}$

\section{Clinical studies (Table I) DAFNE trial}

The Dronedarone Atrial FibrillatioN study after Electrical Cardioversion (DAFNE) was the first double-blind, randomized, placebo controlled trial designed to evaluate the efficacy and safety of dronedarone. ${ }^{15} 270$ patients with persistent AF were randomly allocated to $800,1200,1600 \mathrm{mg}$ daily doses of dronedarone or placebo in 50 centres and 11 countries. The main analysis was conducted on 199/270 patients, who entered the maintenance phase following pharmacological cardioversion or, if unsuccessful, DC cardioversion. The primary outcome was time to first documented AF recurrence. Secondary study endpoints were spontaneous conversion of AF following randomization, heart rate in case of $\mathrm{AF}$ recurrence and the incidence of side effects. 6-month follow-up showed that treatment with dronedarone $800 \mathrm{mg}$ significantly prolonged the time to AF recurrence with a median time to recurrence of 60 days vs 5.3 days in the placebo group (relative risk reduction $55 \%$ [95\% CI, $28 \%$ to $72 \%$ ] $P=0.001$ ).

The incidence of spontaneous conversion to sinus rhythm was associated with a significant dose-effect relationship $(P=0.0261)$. Patients in the dronedarone 800,1200 , and $1600 \mathrm{mg}$ groups exhibited $5.8 \%, 8.2 \%$, and $14.8 \%$ conversion rates respectively, vs $3.1 \%$ on placebo. At the time of first AF recurrence, dronedarone appeared to slow the ventricular response in a dose-dependent fashion. Patients receiving 800,1200 , or $1600 \mathrm{mg}$ dronedarone had their ventricular rate

Table I Summary of clinical trials

\begin{tabular}{|c|c|c|c|c|}
\hline Trial & Subjects enrolled & Follow-up period & Main outcome & Common side effects \\
\hline DAFNE $^{15}$ & 270 & 6 months & $\begin{array}{l}\text { First AF recurrence was } \\
5.8 \% \text { with } 800 \mathrm{mg}, 8.2 \% \text { with } \\
1200 \mathrm{mg} \text { and } 14.8 \% \text { with } \\
1600 \mathrm{mg} \text { dronedarone vs } \\
3.1 \% \text { in placebo }(P=0.026 \mathrm{I})\end{array}$ & Gastrointestinal \\
\hline $\begin{array}{l}\text { EURIDIS } \\
\text { and ADONIS }{ }^{16}\end{array}$ & $\begin{array}{l}612 \text { in EURIDIS } \\
\text { and } 625 \text { in ADONIS }\end{array}$ & 12 months & $\begin{array}{l}\text { First recurrence of AF/AFL } \\
\text { was } 64.1 \% \text { with dronedar- } \\
\text { one vs } 75.2 \% \text { with placebo } \\
(P<0.001)\end{array}$ & Gastrointestinal (diarrhea) \\
\hline ERATO $^{17}$ & 174 & 6 months & $\begin{array}{l}\text { Reduction of II.7 beats per } \\
\text { minute in ventricular rate at } \\
\text { day I4 }(P<0.000 \mathrm{I})-\text { this } \\
\text { effect was sustained for } \\
\text { the duration of trial }(-8.8 \\
\text { beat/minute at } 4 \text { months) } \\
(P<0.00 \mathrm{I})\end{array}$ & $\begin{array}{l}\text { Infections } \\
\text { Mild increase in serum } \\
\text { creatinine levels }\end{array}$ \\
\hline ANDROMEDA $^{18}$ & 627 & $\begin{array}{l}\text { I } 3 \text { months (including } \\
\text { additional } 6 \text { months } \\
\text { after premature } \\
\text { discontinuation } \\
\text { of study) }\end{array}$ & $\begin{array}{l}\text { Premature termination of } \\
\text { trial due to excess mortality } \\
\text { related to the worsening of } \\
\text { heart failure in dronedarone } \\
\text { group (hazard ratio of } 2.13 \text {; } \\
95 \% \mathrm{Cl} 1.07 \text { to } 4.25 ; P=0.03 \text { ) }\end{array}$ & $\begin{array}{l}\text { Worsening heart failure } \\
\text { Increase in serum creatinine } \\
\text { levels }\end{array}$ \\
\hline ATHENA $^{21}$ & 4628 & 21 months & $\begin{array}{l}\text { First hospitalization due } \\
\text { to cardiovascular events } \\
\text { or death was } 31.9 \% \text { in } \\
\text { dronedarone group vs } 39.4 \% \\
\text { in placebo group (hazard } \\
\text { ratio of } 0.76 ; 95 \% \mathrm{Cl} 0.69 \text { to } \\
0.84 ; P<0.00 \mathrm{I} \text { ) }\end{array}$ & $\begin{array}{l}\text { Gastrointestinal (diarrhea, } \\
\text { nausea) Increase in serum } \\
\text { creatinine levels } \\
\text { Rash Bradycardia }\end{array}$ \\
\hline
\end{tabular}

Abbreviations: $\mathrm{AF}$, atrial fibrillation; $\mathrm{AFL}$, atrial flutter; $\mathrm{Cl}$, confidence interval. 
reduced by $13.2,19.2$, and $17.8 \mathrm{bpm}$ on average, respectively, compared to those on placebo $(P=0.0001)$. Twenty-two $(10.8 \%)$ dronedarone patients discontinued treatment due to adverse events. In the $800 \mathrm{mg}, 1200 \mathrm{mg}$ and $1600 \mathrm{mg}$ dronedarone groups, the discontinuation rates were $3.9 \%$, $7.6 \%$ and $22.6 \%$, respectively. The most common cause of drug discontinuation was gastrointestinal side effects. Importantly no evidence of thyroid, ocular, or pulmonary toxicity was observed. There were no proarrhythmic reactions. Drug-induced QT prolongation was noticed only in the $1600 \mathrm{mg}$ group and the effect of dronedarone on the QT interval remained modest. Dronedarone also proved to be hemodynamically well tolerated. Thus the DAFNE trial showed that dronedarone, at an $800 \mathrm{mg}$ daily dose, appears to be effective and safe for the prevention of AF relapses after cardioversion at a 6-month follow-up.

\section{EURIDIS and ADONIS trials}

The results of DAFNE provided promising role of dronedarone (400 $\mathrm{mg}$ bid) in prevention of AF recurrence and later it was evaluated by two similar double-blind, placebocontrolled, multicenter, multinational, pivotal phase III studies with similar study protocols to determine the efficacy of dronedarone (400 mg bid) for the maintenance of normal sinus rhythm after electrical, pharmacological, or spontaneous conversion of AF or AFL (atrial flutter). The European Trial in Atrial Fibrillation or Flutter Patients Receiving Dronedarone for the Maintenance of Sinus Rhythm (EURIDIS) was carried out at 65 centers in 12 European countries and the American-Australian-African Trial with Dronedarone in Atrial Fibrillation/Flutter Patients for the Maintenance of Sinus Rhythym (ADONIS) at 101 centers in USA, Canada, Australia, South Africa and Argentina. ${ }^{16}$

Male and female patients over 21 years of age were enrolled provided they had at least one episode of AF (as seen on electrocardiography) in the preceding 3 months, and were in sinus rhythm for at least 1 hour before randomization. Patients previously treated with amiodarone were permitted, and were enrolled immediately after its discontinuation. Eligible patients $(n=612$ in EURIDIS and $n=625$ in ADONIS) were randomly assigned in a $2: 1$ ratio to receive either $400 \mathrm{mg}$ of oral dronedarone twice daily or a matching placebo for 1 year. Follow-up visits were scheduled for review of symptoms, assessment of vital signs, and performance of electrocardiography on days 7, 14, and 21 and at $2,4,6,9$, and 12 months. The primary endpoint was the time to the first documented recurrence of $\mathrm{AF} / \mathrm{AFL}$, defined as an episode lasting for at least 10 minutes and confirmed by two consecutive recordings on 12-lead electrocardiography or Tran telephonic monitoring. The secondary endpoints were severity of symptoms related to AF/AFL during recordings of 12-lead electrocardiography or Tran telephonic monitoring and the mean ventricular rate during the first recurrence.

In EURIDIS, the median time to relapse was 96 days in the dronedarone group and 41 days in the placebo group and in ADONIS it was 158 days in the dronedarone group and 59 days in the placebo group. For the two trials combined, the median times to recurrence were 116 days in the dronedarone group and 53 days in the placebo group. Combining data for the two trials, at 12 months, the rates of recurrence were $64.1 \%$ in the dronedarone group and $75.2 \%$ in the placebo group (hazard ratio, $0.75 ; 95 \% \mathrm{CI}, 0.65$ to 0.87 ; $P<0.001)$. When the data for the two trials were analyzed separately, the results were similar with reductions in the risk of AF/AFL recurrence over 1 year by $29 \%(P=0.0059)$ and $26 \%(P=0.0244)$ in EURIDIS and ADONIS, respectively. Mean ventricular rate during first AF/AFL recurrence was 102 and $104 \mathrm{bpm}$ in the dronedarone group of EURIDIS and ADONIS, respectively, whereas the the corresponding rates in the placebo groups were 117 and $116 \mathrm{bpm}$, respectively ( $P<0.001$ and $P<0.001$, respectively). Combining the pooled data from trials, $37.7 \%$ of patients in the dronedarone group and $46.0 \%$ of patients in the placebo group had symptomatic recurrences $(P<0.001$ by the log-rank test). In both trials, dronedarone was superior to placebo in variety of patient subgroups including hypertension, structural heart disease, and previous amiodarone use.

There was no evidence of pulmonary or thyroid toxicity in dronedarone group. The incidence of hyperthyroidism in dronedarone group $(8.4 \%)$ was significantly lower than in placebo group $(14.1 \%)(P=0.002)$, and the incidence of hypothyroidism $(5.5 \%)$ in dronedarone group was slightly higher than in placebo group $(3.5 \%)$ but not significantly $(P=0.15)$. The incidence of photosensitive rash and reaction was not significant $(0.7 \%$ in dronedarone group vs $0.2 \%$ in placebo group $[P=0.44])$. The main side effect of dronedarone group was diarrhea at high doses. In addition, there was a higher incidence of elevated serum creatinine levels in the dronedarone group than in the placebo group $(2.4 \%$ vs $0.2 \%, P=0.004)$. Serious side effects were rare and were reported with similar frequency in both groups (16.5\% dronedarone vs $13.5 \%$ placebo). In the combined European and non-European trials, dronedarone was safe in maintaining sinus rhythm without major prolongation of either the QT or the QTc interval (lengthening by 23 and 9 msec, respectively, without significant effects on the QRS 
duration) with no reports of torsades. When analyzed separately or in combination, dronedarone proved to be superior in maintaining sinus rhythm with an excellent safety and tolerability profile.

\section{ERATO trial}

The Efficacy and safety of dRonedArone for The cOntrol of ventricular rate during atrial fibrillation (ERATO) trial assessed the efficacy of dronedarone in the control of ventricular rate in patients with permanent AF, when added to standard rate-control therapy. ${ }^{17}$ In this prospective, multinational, double-blind, randomized study, 174 elderly patients with permanent AF were randomized to 6 months' treatment with dronedarone, $400 \mathrm{mg}$ twice a day $(\mathrm{n}=85)$ or matching placebo $(n=89)$. All patients were receiving standard rate-control therapy beta-blockers, calcium antagonists, and digoxin. In this study, 38.9\% had structural heart disease and $39.7 \%$ had NYHA class I or II heart failure. The primary endpoint was the change in mean ventricular rate between baseline and day 14, as assessed by 24-hour Holter. Ventricular rate was also assessed during submaximal and maximal exercise.

At day 14, mean reduction in mean 24-hour ventricular rate in the dronedarone group was $11.0 \mathrm{bpm}$ as opposed to an increase of $0.7 \mathrm{bpm}$ in the placebo group, a treatment effect of $-11.7 \mathrm{bpm}(P<0.0001)$. Comparable reductions were sustained throughout the 6-month trial. During maximal exercise and compared to placebo, there was a mean reduction of $24.5 \mathrm{bpm}(P<0.0001)$, without any reduction in exercise tolerance as measured by maximal exercise duration. The effects of dronedarone were additive to those of other rate-control agents, including beta-blockers, calcium antagonists, and digoxin. Thus, dronedarone appears to improve ventricular rate control in patients with permanent AF without any organ toxicity or proarrhythmia.

In the ERATO study, there was a mean $41.4 \%$ increase in digoxin levels in patients taking concomitant dronedarone, but without significant difference between the treatment groups in number of patients with an increase in digoxin outside the normal range $(4.5 \%$ in the dronedarone group vs $2.8 \%$ in the placebo group). Also dronedarone had no effect on international normalized ratio in patients taking oral anticoagulants.

\section{ANDROMEDA trial}

In the EURIDIS and ADONIS trials, patients with NYHA class III and IV were excluded. The Antiarrhythmic Trial with Dronedarone in Moderate to Severe congestive heart failure Evaluating Morbidity Decrease (ANDROMEDA) study was double-blind, placebo-controlled, randomized in 1:1 ratio, parallel-group trial comparing treatment with $400 \mathrm{mg}$ of dronedarone twice daily with administration of matching placebo to assess the rate of hospitalization and mortality in patients with heart failure. ${ }^{18}$ Patients with symptomatic heart failure (NYHA class III or IV) requiring diuretic treatment, or paroxysmal nocturnal dyspnea within the month before admission with a left ventricular ejection fraction of $<35 \%$ were enrolled. The study began in June 2002 and was originally scheduled to last for 2 years, and each patient was to be treated with dronedarone or matching placebo for a minimum of 12 months. However, in January 2003, the trial was terminated owing to an excess of deaths in the dronedarone group. Follow-up was continued until at least 6 months after withdrawal of the study drug. 627 patients had been enrolled (310 in dronedarone group and 317 in placebo group) and treated by the time the trial was stopped at a median follow-up of 2 months. During this period, 25 patients in the dronedarone group (8.1\%) and 12 patients in the placebo group (3.8\%) died (hazard ratio in the dronedarone group, 2.13; $95 \%$ CI 1.07 to $4.25 ; P=0.03$ ). The excess mortality was predominantly due to worsening of heart failure (10 deaths in the dronedarone group and 2 in the placebo group). After 6 months of discontinuation of treatment, the mortality rates were comparable in both groups, 42 patients in the dronedarone group (13.5\%) and 39 patients in the placebo group (12.3\%) (hazard ratio, $1.13 ; 95 \% \mathrm{CI}, 0.73$ to 1.74 ; $P=0.60$ ). The primary endpoint (death from any cause or hospitalization for worsening heart failure) did not differ significantly between the two groups; there were 53 events in the dronedarone group (17.1\%) and 40 events in the placebo group (12.6\%) (hazard ratio, 1.38; 95\% CI, 0.92 to $2.09 ; P=0.12)$.

In the ANDROMEDA trial, the estimated glomerular filtration rate (EGFR) was decreased early after study treatment was started in patients who received dronedarone. Mean EGFR was $7 \mathrm{~mL}$ per minute per $1.73 \mathrm{~m}^{2}$ of body-surface area lower in the dronedarone group than in the placebo group $(P=0.009)$. After treatment with the study drug was terminated, EGFR returned to baseline values. However, the fall in EGFR may not reflect a deterioration of renal function. Dronedarone has been shown to reduce creatinine clearance by about $18 \%$, with no evidence of an effect on the measured (as opposed to estimated) GFR, suggesting that dronedarone causes a specific partial inhibition of tubular organic cation transporters. ${ }^{19}$ 
There was no evidence of proarrhythmia or an increased incidence of sudden death in the dronedarone group. Retrospective analysis of ANDROMEDA revealed an inappropriate discontinuation of angiotensin-converting enzyme inhibitor (ACEI)/angiotensin receptor blocker (ARB) in the dronedarone group after transient rise in serum creatinine levels early after the initiation of dronedarone. It is suspected that inappropriate discontinuation of $\mathrm{ACEI} / \mathrm{ARB}$ with subsequent pump failure may be a contributing factor for excess mortality. Other proposed explanations included negative ionotropic effect or the play of chance but the precise explanation is still not clear.

Of note, ANDROMEDA was not an AF study. A history or the presence of AF was not an inclusion criterion for this study. A history of AF (chronic, persistent, or paroxysmal) was reported in almost $40 \%$ of the patients, whereas AF at the time of randomization was present in $23.2 \%$ of the patients in the dronedarone group and $26.8 \%$ in the placebo group. Given the results of ANDROMEDA, dronedarone is not recommended for patients with NYHA class IV symptoms or cardiogenic shock.

\section{ATHENA trial}

Post hoc analysis from the EURIDIS and ADONIS trial showed that dronedarone reduced the risk of all-cause hospitalization or death by $27 \%$ compared to placebo $(95 \%$ CI $7 \%$ to $43 \% ; \mathrm{P}=0.01) .{ }^{20}$ To elucidate this potential clinical benefit, the ATHENA (A Trial to Assess the Efficacy of Dronedarone for the Prevention of Cardiovascular Hospitalization or Death from any cause in Patients with Atrial Fibrillation/Atrial Flutter) was conducted recently. ${ }^{21}$ It was a prospective, randomized, placebo-controlled, double-blind, multi-national, multi-center, parallel-group trial evaluating the effects of dronedarone $400 \mathrm{mg}$ bid versus placebo (ratio 1:1) in patients with paroxysmal or persistent AF/AFL who had additional risk factors for death. 4628 patients were enrolled, 2301 received dronedarone and 2327 received placebo. The mean follow-up period was 21 months.

ATHENA was the landmark study that evaluated for the first time a treatment on the top of standard background therapy for the management of AF in reducing morbidity and mortality by preventing cardiovascular hospitalizations or death from any cause. It was the largest study ever conducted in a typical AF population (4628 patients were enrolled). The primary outcome was the first hospitalization due to cardiovascular events or death. This was the first large trial where a rhythm-control agent was studied with this primary endpoint in patients with AF/AFL. Secondary outcomes were death from any cause, death from cardiovascular causes, and hospitalization due to cardiovascular events. Patients enrolled in this study had one of the following risk factors: age overt 70 years; arterial hypertension; diabetes mellitus; previous stroke or systemic embolism; left atrial enlargement; or left ventricular ejection fraction $\leq 40 \%$. Therefore, the patients enrolled in ATHENA represent the largest group of individuals seeking medical attention for treatment of AF. Patients were ineligible for participation in ATHENA if they had one of the following cardiac conditions: permanent AF, unstable hemodynamic situation (ie, recently decompensated heart failure), or congestive heart failure NYHA class IV.

In the dronedarone group $734(31.9 \%)$ had a primary outcome event and in the placebo group, 917 patients $(39.4 \%)$ had a primary outcome event. The hazard ratio for the primary outcome in the dronedarone group was 0.76 (95\% CI, 0.69 to $0.84 ; P<0.001$ ). There were 116 deaths $(5.0 \%)$ in the dronedarone group and $139(6.0 \%)$ in the placebo group (hazard ratio, $0.84 ; 95 \% \mathrm{CI}, 0.66$ to 1.08 ; $P=0.18)$. In the dronedarone group 63 patients died from cardiovascular causes $(2.7 \%)$ while $90(3.9 \%)$ patients died in the placebo group (hazard ratio, $0.71 ; 95 \% \mathrm{CI}, 0.51$ to 0.98 ; $P=0.03)$, largely due to a reduction in the rate of death from arrhythmia with dronedarone. There were 26 deaths from cardiac arrhythmia (in $1.1 \%$ of patients) in the dronedarone group and 48 (in 2.1\%) in the placebo group (hazard ratio, $0.55 ; 95 \% \mathrm{CI}, 0.34$ to $0.88 ; P=0.01)$.

675 patients $(29.3 \%)$ in the dronedarone group had a first hospitalization due to cardiovascular events compared with 859 patients $(36.9 \%)$ in the placebo group (hazard ratio, $0.74 ; 95 \% \mathrm{CI}, 0.67$ to $0.82 ; P<0.001)$. This reduction in the rate of hospitalization due to cardiovascular events was mainly due to reduction in the number of hospitalizations for AF. Also hospitalization due to any cardiovascular event or death from any cause was less in the dronedarone group (1253 patients $(54.5 \%))$ vs 1668 patients $(71.7 \%)$ in the placebo group (hazard ratio, $0.76 ; 95 \%$ CI, 0.68 to $0.84 ; P<0.001)$. Total hospital nights spent by the patients in the dronedarone group were significantly less than in the placebo group (9995 vs 13986 ; $P<0.001)$. This reduction of almost 4000 hospital days translates to a decrease of 1.26 hospitalization days per patient per year. In patients with permanent AF there was $26 \%$ drop in risk of cardiovascular hospitalization or death. They had a similar hazard ratio for the primary endpoint to the overall study population. ${ }^{22}$

The study drug was prematurely discontinued in 696 $(30.2 \%)$ of patients receiving dronedarone and in $716(30.8 \%)$ 
of those receiving placebo. The main reasons were adverse events. Bradycardia, QT-interval prolongation, diarrhea, nausea, rash, and an increase in the serum creatinine level were significantly more common in the dronedarone group than in the placebo group. No significant increase in the rates of thyroid or pulmonary disorders was seen with dronedarone. The most frequently reported adverse events were gastrointestinal: $26 \%$ in dronedarone vs $22 \%$ in placebo.

The ATHENA trial concluded that treatment with dronedarone significantly reduces the incidence of hospitalization due to cardiovascular events or death in patients with paroxysmal or persistent AF/AFL. In a post-hoc analysis of the ATHENA trial, dronedarone was associated with a $34 \%$ decrease in adjusted risk of stroke compared with placebo over a follow-up averaging 21 months $(1.19 \%$ per year in dronedarone group vs $1.79 \%$ per year in placebo group) (hazard ratio, $0.66 ; 95 \% \mathrm{CI}, 0.46$ to $0 ; P=0.027$ ). ${ }^{23}$

The ANDROMEDA study enrolled only patients with advanced heart failure and recent decompensation leading to hospitalization while patients with severe (NYHA class IV) heart failure were excluded in ATHENA. Given the results of both trials it is postulated that dronedarone increases cardiovascular mortality among patients with advanced and recently decompensated congestive heart failure but reduces cardiovascular mortality in patients with less severe heart failure.

On the basis of ANDROMEDA study, dronedarone failed in its first appearance before the US Food and Drug Administration (FDA) committee in August 2006. However, after the results of ATHENA study, dronedarone was resubmitted to the FDA and the European Medicines Agency for regulatory panel. On March 18, 2009 it was recommended by an Advisory Committee of the US FDA for approval in the management of AF. The FDA approved dronedarone $400 \mathrm{mg}$

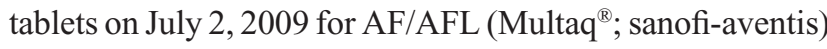
with a boxed warning that the drug should not be used in severe heart failure patients.

\section{DIONYSUS trial}

In the Efficacy and Safety of Dronedarone vs Amiodarone for the maintenance of Sinus Rhythm in Patients with AF (DIONYSUS) trial, dronedarone was compared with amiodarone for the safety and efficacy in the maintenance of sinus rhythm in patients with AF (not AFL). 500 patients were involved in the study and had a follow-up of 6 months. Dronedarone was less effective for the maintenance of sinus rhythm. Rate of recurrent AF was 63\% with dronedarone and $42 \%$ with amiodarone. But the use of dronedarone was associated with fewer side effects and less premature discontinuation of drug treatment. ${ }^{24}$

\section{Conclusions}

Dronedarone is a newer benzofuran derivative structurally similar to amiodarone except it lacks the iodine moiety and there is an addition of a methane sulfonyl group. It does not significantly prolong the QTc and no significant pulmonary, hepatic, ocular, or neurologic toxic effects have been observed in the studies done to date. Dronedarone, in addition to its benefits for rate and rhythm control, may reduce all-cause hospitalization or death in patients with AF. Dronedarone may be a viable, uniformly effective, and safer treatment option for patients with AF.

\section{Disclosures}

The authors declare no conflicts of interest.

\section{References}

1. Go AS, Hylek EM, Phillips KA, et al. Prevalence of diagnosed atrial fibrillation in adults: national implications for rhythm management and stroke prevention: the AnTicoagulation and Risk Factors in Atrial Fibrillation (ATRIA) Study. JAMA. 2001;285(18):2370-2375.

2. Singh BN, Singh SN, Reda DJ, et al. Sotalol Amiodarone Atrial Fibrillation Efficacy Trial (SAFE-T) Investigators. Amiodarone versus sotalol for atrial fibrillation. $N$ Engl J Med. 2005;352(18):1861-1872.

3. Hohnloser S, Klingenheben T, Singh BN. Amiodarone-associated proarrhythmic effects: a review with special reference to torsade de pointes tachycardia. Ann Intern Med. 1994;121:529-535.

4. Han TS, Williams GR, Vanderpump MP. Benzofuran derivatives and the thyroid. Clin Endocrinol (Oxf). 2009;70(1):2-13.

5. Van Beeren HC, Jong WM, Kaptein E, et al. Dronerarone acts as a selective inhibitor of 3,5,3'-triiodothyronine binding to thyroid hormone receptor-alpha1: in vitro and in vivo evidence. Endocrinology. 2003;144(2):552-558

6. Sun W, Sarma JS, Singh BN. Electrophysiological effects of dronedarone (SR33589), a noniodinated benzofuran derivative, in the rabbit heart: comparison with amiodarone. Circulation. 1999;100(22): 2276-2281.

7. Varro A, Takacs J, Nemeth M, et al. Electrophysiological effects of dronedarone (SR 33589), a noniodinated amiodarone derivative in the canine heart: comparison with amiodarone. $B J$ Pharmacol 2001;133:625-634.

8. Gautier P, Guillemare E, Marion A, et al. Electrophysiologic characterization of dronedarone in guinea pig ventricular cells. $J$ Cardiovasc Pharmacol. 2003;41(2):191-202.

9. Lalevee N, Nargeot J, Barrere-Lemaire S, et al. Effects of amiodarone and dronedarone on voltage-dependent sodium current in human cardiomyocytes. J Cardiovasc Electrophysiol. 2003;14(8):885-890.

10. Guillemare E, Marion A, Nisato D, et al. Inhibitory effects of dronedarone on muscarinic $\mathrm{K}+$ current in guinea pig atrial cells. $J$ Cardiovasc Pharmacol. 2000;36(6):802-805.

11. Hodeige D, Heyndrickx JP, Chatelain P, et al. SR 33589, a new amiodarone-like antiarrhythmic agent: anti-adrenoceptor activity in anaesthetized and conscious dogs. European Journal of Pharmacology. 1995;279(1):25-32.

12. Djandjighian L, Planchenault J, Finance O, et al. Hemodynamic and antiadrenergic effects of dronedarone and amiodarone in animals with a healed myocardial infarction. $J$ Cardiovasc Pharmacol. 2000;36(3):376-383. 
13. Manning AS, Bruyninckx C, Ramboux J, et al. SR 33589, a new amiodarone-like agent: effect on ischemia- and reperfusioninduced arrhythmias in anesthetized rats. $J$ Cardiovasc Pharmacol. 1995;26(3):453-461.

14. Finance O, Manning A, Chatelain P. Effects of a new amiodarone-like agent, SR 33589, in comparison to amiodarone, D,L-sotalol, and lignocaine, on ischemia-induced ventricular arrhythmias in anesthetized pigs. J Cardiovasc Pharmacol. 1995;26(4):570-576.

15. Touboul P, Brugada J, Capucci A, et al. Dronedarone for prevention of atrial fibrillation: A dose-ranging study. Eur Heart J. 2003;24: 1481-1487.

16. Singh BN, Connolly SJ, Crijns HJ, et al. Dronedarone for Maintenance of Sinus Rhythm in Atrial Fibrillation or Flutter. N Engl J Med. 2007;357(10):987-999.

17. Davy JM, Herold M, Hoglund C, et al. Dronedarone for the control of ventricular rate in permanent atrial fibrillation: the Efficacy and safety of dRonedArone for the cOntrol of ventricular rate during atrial fibrillation (ERATO) study. Am Heart J. 2008;156(3):527. e1-e9.

18. Kober L, Torp-Pedersen C, McMurray JJ, et al. Dronedarone Study Group Increased mortality after dronedarone therapy for severe heart failure. N Engl J Med. 2008;358(25):2678-2687.
19. Tschuppert Y, Buclin T, Rothuizen LE, et al. Effect of dronedarone on renal function in healthy subjects. Br J Clin Pharmacol. 2007;64: 785-791.

20. Hohnloser SH, Goethe KD, Singh BN. Dronedarone Significantly Decreases the Combined Endpoint of Hospitalization and Death in Patients with Atrial Fibrillation. Results from the EURIDIS/ADONIS study. American Heart Association 2005 Scientific Sessions; November 13-16, 2005; Dallas Convention Center - Dallas, TX. Abstract 1637.

21. Hohnloser SH, Crijns HJ, van Eickels M, et al. Effect of dronedarone on cardiovascular events in atrial fibrillation. $N$ Engl J Med. 2009;360(7):668-678.

22. Torp-Pedersen C, Page RL, Connolly SJ, et al. The effect of dronedarone on hospitalizations in patients with atrial fibrillation. Results from the ATHENA study. American Heart Association 2008 Scientific Sessions; November 8-12, 2008; New Orleans, LA. Abstract 4101.

23. Connolly SJ. ATHENA: The effect of dronedarone on cardiovascular outcomes and stroke in patients with atrial fibrillation. European Society of Cardiology Congress 2008; September 3, 2008; Munich, Germany. Clinical trials update 3 .

24. Zimetbaum PJ. Dronedarone for atrial fibrillation - an odyssey. N Engl J Med. 2009;360(18):1811-1813.
Vascular Health and Risk Management

\section{Publish your work in this journal}

Vascular Health and Risk Management is an international, peerreviewed journal of therapeutics and risk management, focusing on concise rapid reporting of clinical studies on the processes involved in the maintenance of vascular health; the monitoring, prevention and treatment of vascular disease and its sequelae; and the involvement of

\section{Dovepress}

metabolic disorders, particularly diabetes. This journal is indexed on PubMed Central and MedLine. The manuscript management system is completely online and includes a very quick and fair peer-review system, which is all easy to use. Visit http://www.dovepress.com/ testimonials.php to read real quotes from published authors. 